\title{
BLNet: Bidirectional learning network for point clouds
}

\author{
Wenkai Han ${ }^{1}$, Hai $\mathbf{W u}^{1}$, Chenglu Wen ${ }^{1}(\bowtie)$, Cheng Wang ${ }^{1}$, and $\mathrm{Xin}_{\mathbf{L i}^{2}}$ \\ (c) The Author(s) 2021.
}

\begin{abstract}
The key challenge in processing point clouds lies in the inherent lack of ordering and irregularity of the 3D points. By relying on perpoint multi-layer perceptions (MLPs), most existing point-based approaches only address the first issue yet ignore the second one. Directly convolving kernels with irregular points will result in loss of shape information. This paper introduces a novel point-based bidirectional learning network (BLNet) to analyze irregular 3D points. BLNet optimizes the learning of $3 \mathrm{D}$ points through two iterative operations: featureguided point shifting and feature learning from shifted points, so as to minimise intra-class variances, leading to a more regular distribution. On the other hand, explicitly modeling point positions leads to a new feature encoding with increased structure-awareness. Then, an attention pooling unit selectively combines important features. This bidirectional learning alternately regularizes the point cloud and learns its geometric features, with these two procedures iteratively promoting each other for more effective feature learning. Experiments show that BLNet is able to learn deep point features robustly and efficiently, and outperforms the prior state-of-the-art on multiple challenging tasks.
\end{abstract}

Keywords point clouds; irregularity; shape features; bidirectional learning

\section{Introduction}

3D point cloud understanding is critical in many realworld vision applications such as autonomous driving,

1 School of Informatics, Xiamen University, 422 Siming South Road, Xiamen 361005, China. E-mail: W. Han, hlxwk@stu.xmu.edu.cn; H. Wu, wuhai@stu.xmu.edu.cn; C. Wen, clwen@xmu.edu.cn (凶); C. Wang, cwang@xmu.edu.cn.

2 School of Electrical Engineering and Computer Science, Louisiana State University, Baton Rouge, LA 70803, USA. E-mail: xinli@cct.lsu.edu.

Manuscript received: 2021-03-12; accepted: 2021-10-05 robotics, and augmented reality. A key challenge for effectively learning point cloud features is that point clouds captured by depth cameras or LiDAR sensors are often unordered and irregular, so many effective deep learning architectures, e.g., as in Refs. [1-3] are not directly applicable.

To tackle this, many approaches convert irregular point clouds into regular data formats such as multiview images [4-6] or 3D voxels [7-11]. However, such conversion processes result in loss of geometric detail and have large memory requirements. Alternatively, some recent studies focus on directly processing point clouds. A seminal approach, PointNet [12], individually learns per-point features using shared MLPs and gathers a global representation with maxpooling. Although effective, this design ignores local structures that constitute the semantics of the whole object. To solve this problem, many subsequent approaches [13-18] partition the point cloud into nested subsets, and then build a hierarchical framework to learn contextual representations from local to global. Nevertheless, these methods operate directly on raw point clouds, whose spatial irregularity limits the methods' inductive learning ability.

3D acquisition typically produces irregular and nonuniformly distributed raw point clouds. Figure 1(b) provides an example of irregular points sampled from a square. Suppose we have shared MLPs $\mathcal{G}$ together with their learnable weights $\boldsymbol{W}$. If we apply these convolutions to the points in Fig. 1, then the convolutional output is $\boldsymbol{f}_{b}=\mathcal{G}\left(\left[p_{1}, p_{2}, p_{3}, p_{4}\right]_{x}, \boldsymbol{W}\right)$, where $x=(a, b)$. The shared point-wise MLPs utilized for encoding points can ensure permutationinvariance and address the lack of ordering. However, due to the irregular sampling in (b), we usually get $\boldsymbol{f}_{a} \neq \boldsymbol{f}_{b}$. Therefore, local features extracted on noisy or irregularly sampled points are often unstable, causing loss of shape information. We observation that: (i) as 


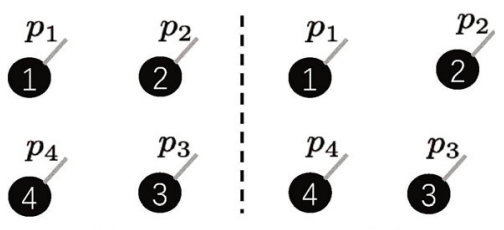

(a)

(b)

Fig. 1 Points in (a) are ideally regularly sampled and while in (b) are irregularly sampled. Each point is associated with coordinates $p_{i}$.

the sampling of (b) becomes more regular, its learned feature will converge to $f_{a}$ and become more stable, but on the other hand (ii) if a more accurate feature description (e.g., $\boldsymbol{f}_{a}$ ) is available, deforming the shape (e.g., shape (b)) according to this feature will allow the point cloud to adjust its points towards a more regular distribution. Thus, making sampled points more regular and obtaining more accurate features are both important issues to address.

Thus, we formulate BLNet, the first work to apply bidirectional learning to point clouds and analyze irregular 3D points through bidirectional interaction between points and features. The key to BLNet is to address two issues iteratively: featureguided point shifting and feature learning from shifted points. On the one hand, taking the task loss as feedback, we use a position feedback module associated with adaptive 3D displacements to automatically adjust the positions of points. By minimizing intra-class variances, 3D points are regularized towards a certain distribution that fits the network well. On the other hand, we use a new feature modeling module, which explicitly encodes point positions with increased structure-awareness. We further use attention pooling to selectively focus upon and combine important features. This bidirectional learning approach alternately regularizes the point cloud and learns its geometric features; these two procedures iteratively support each other to provide more effective feature learning. Extensive experiments have verified the superiority of our BLNet on multiple challenging datasets including ModelNet40 [8], ShapeNet Parts [19], S3DIS [20], and ScanNet [9]. Moreover, we show ablation experiments and visual results to provide a better understanding of BLNet.

\section{Related work}

\subsection{Deep learning on regular domain}

To leverage the impressive success of traditional convolution on regular data representations (e.g., images), many approaches transform irregular point clouds into regular data formats such as multi-view images and 3D voxels. In the former, view-based methods [4-6] render multiple images from point clouds from different viewpoints and apply standard CNNs to the rendered images. In the latter, voxelbased methods [7-11] structure the point cloud using $3 \mathrm{D}$ regular voxels. Afterwards, 3D CNNs can be directly applied as to images. However, these regular formats need projection or voxelization, which result in a quantifiable loss of geometric information. By contrast, our BLNet directly processes point clouds and does not rely on any transformations.

\subsection{Deep learning on irregular domains}

Inspired by the pioneering works on PointNet $[12,13]$, many recent methods directly process point clouds, using sophisticated networks to capture features. These approaches can be generally classified as using (i) neighbouring feature pooling [13, 15, 18, 21, 22], (ii) graph message passing [16, 23, 24], (iii) kernel-based convolution [14, 25-28], or (iv) transformer-based learning $[29,30]$. These methods directly run on raw point clouds. The spatial irregularity of point clouds limits their inductive learning ability.

\subsection{Bidirectional learning}

Bidirectional learning has been shown effective, with enhanced performance over uni-directional learning in multiple tasks such as language translation [31, 32], image generation [33], and image translation [34]. It utilizes additional top-down (target-to-source) training to reduce the uni-directional dependency between source and target. However, such methods typically train the two directions separately and fuse them afterwards. In contrast, our BLNet is the first work to provide bidirectional interaction between points and features on point clouds. It alternately combines two learning directions, consequently forming a complete network for training.

\section{Our method}

\section{$3.1 \quad$ Locality}

Most recent point cloud learning frameworks [13, 14, 18] are trained to extract representations based on local features, which has been shown more effective than earlier work [12] that learns global descriptions. Thus, we design our network and modules to work locally. 


\subsection{Bidirectional learning}

The position feedback module performs featureguided point shifting towards a more regular distribution, while the feature modeling module explores discriminative features from shifted points. To realize our bidirectional learning pipeline, we integrate a position feedback module and a feature modeling module into one bidirectional convolution operator, namely, BidConv. Stacking multiple BidConv operations enables the two modules to execute alternately and assist each other, as illustrated in Fig. 2.

\subsection{BLNet architecture}

As shown in Fig. 2, we have devised a hierarchical framework, BLNet, which can be applied to multiple tasks including point cloud classification and segmentation. In both tasks, we use four BidConv units to learn dimension-increasing features with progressive downsampling. The final global representation is followed by fully connected layers for classification tasks. For segmentation tasks, highresolution point-wise predictions are required, and this can be realized by deconvolution; we still utilize BidConv to recover resolution, and progressively upsample the compact features obtained from the encoder to the original resolution. Higher-resolution points are forwarded from earlier corresponding convolution layers, following the coarse-to-fine design of U-Net. Inspired by Ref. [35], features at the same resolution are skip-connected to preserve previous information. Both classification and segmentation models can be trained in an end-to-end manner.

\subsection{Feature modeling}

In order to learn more accurate features, and use them to guide the shifting of points, we have developed a new feature modeling module. This module includes a position encoding unit and an attention pooling unit, which can more discriminatively capture and combine features.

\subsubsection{Position encoding}

Given a point cloud $P$ together with corresponding point features (e.g., raw RGB, or intermediate learned features), this unit aims to explicitly encode the spatial layout of 3D points, which plays a crucial role in shape analysis. Existing approaches [13, 15, 16] typically concatenate position information with point features, and then transform the concatenated results for feature learning. But these approaches are suboptimal at capturing meaningful geometry patches. In contrast, we perform an explicit encoding of point positions first and then combine the output with point features for further enhancement. This enables each 3D point to observe its local geometry, thus eventually enriching the entire network with

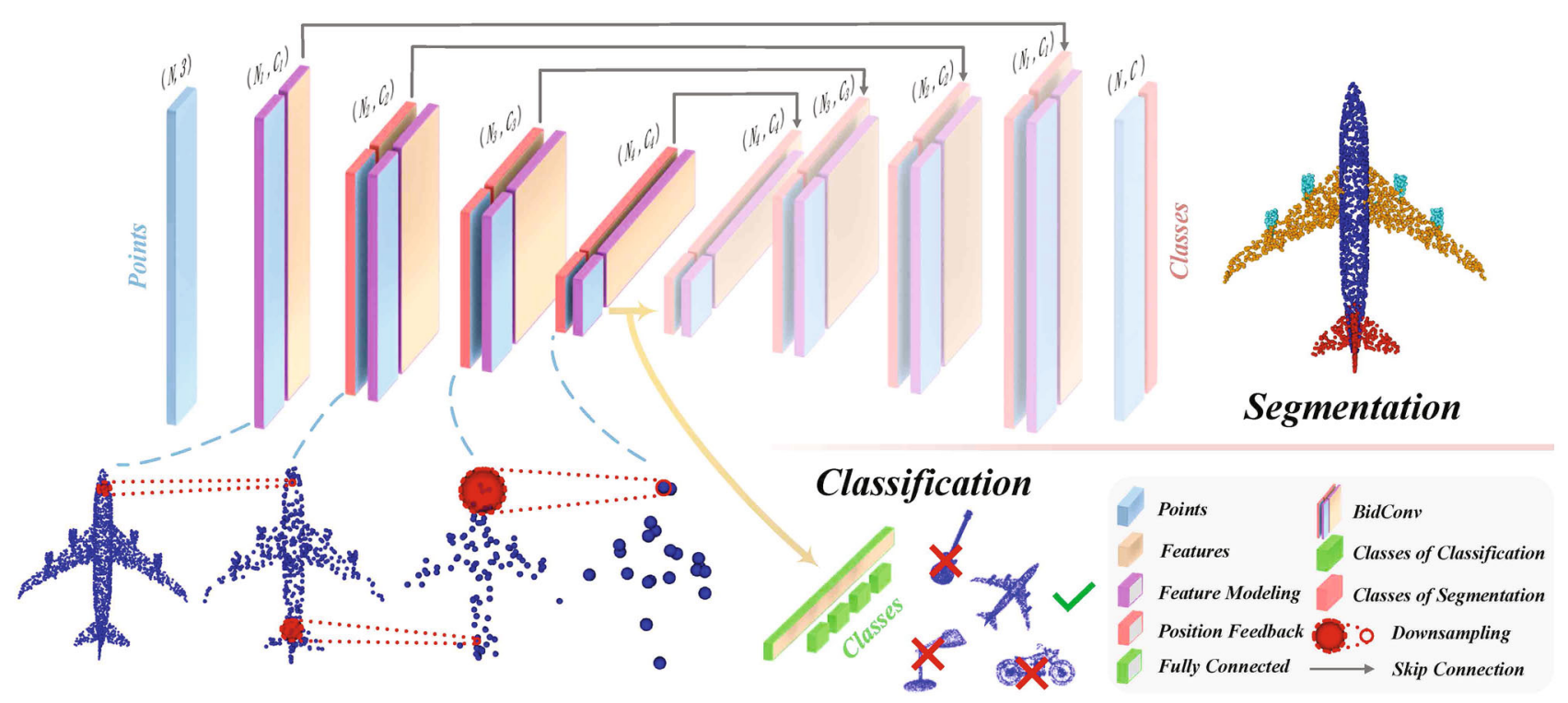

Fig. 2 BLNet architecture. Classification uses 4 BidConv before the fully connected classifier. Segmentation follows a U-net architecture. Points downsampling (using FPS [13]) and upsampling are also included in convolution, depending on its use. $N_{1}>N_{2}>N_{3}>N_{4}$ indicates points downsampling in each convolution, and $C_{1}<C_{2}<C_{3}<C_{4}$ denotes dimension-increasing feature channels at each point. The first BidConv does not include a position feedback module, and no features extracted from points exist at the beginning of the network. 
increased structure-awareness. Particularly, this unit consists of the following steps:

Localisation. For the $i$-th point, to increase its receptive field, we index its neighboring points with a dilated $K$-nearest neighbours (KNN) algorithm. Specifically, we sample $K$ points at equal intervals from the top $K \times r$ neighboring points, where $r$ denotes the dilation rate.

Position encoding. For each of the $K$ neighboring points $\left\{p_{i}^{1} \ldots p_{i}^{k} \ldots p_{i}^{K}\right\}$ of the center point $p_{i}$, we explicitly encode their relative position as Eq. (1):

$$
\boldsymbol{r}_{i}^{k}=\mathcal{G}_{r}\left(p_{i}^{k}-p_{i}, \boldsymbol{W}_{r}\right)
$$

where $p_{i}$ and $p_{i}^{k}$ are global $(x, y, z)$ coordinates of points, and $p_{i}^{k}-p_{i}$ gives relative coordinates, providing translation invariance. $\mathcal{G}_{r}()$ is a shared function, with learnable weights $\boldsymbol{W}_{r}$. This function can be implemented with any differentiable architecture, and we use point-wise shared MLPs in this work to address the lack of ordering of $3 \mathrm{D}$ points. Note that all the functions in our paper are complemented by per-point MLPs, and we omit explaining this in the remaining of this paper.

Feature enhancement. The prior semantic information contained in per-point features can further enhance the distinctiveness of learned position features. For each neighboring point $p_{i}^{k}$, we concatenate its position features $\boldsymbol{r}_{i}^{k}$ with corresponding point features $\boldsymbol{f}_{i}^{k}$, and then use a shared function $\mathcal{G}_{f}()$ to combine them. Thus, we obtain the enhanced feature vector $\hat{\boldsymbol{f}}_{i}^{k}$ with the following formulation:

$$
\hat{\boldsymbol{f}}_{i}^{k}=\mathcal{G}_{f}\left(\left[\boldsymbol{r}_{i}^{k}, \boldsymbol{f}_{i}^{k}\right], \boldsymbol{W}_{f}\right)
$$

where [,] is the concatenation operation.

\subsubsection{Attention pooling}

This unit is used to aggregate the set of neighboring point features. Different features in the local region have varied impacts on the local representation. The leading strategy for integrating the neighboring features in existing work $[13,16]$ is max/mean pooling. However, this frequently results in a loss of useful information. In contrast, our new attention pooling unit selectively focuses on the most relevant features In particular, this unit includes the following steps:

Attention scoring. Given the set of local features $\hat{\boldsymbol{F}}_{i}=\left\{\hat{\boldsymbol{f}}_{i}^{1} \ldots \hat{\boldsymbol{f}}_{i}^{k} \ldots \hat{\boldsymbol{f}}_{i}^{K}\right\}$, our shared function $\mathcal{G}_{s}()$ learns a unique attention score for each channel of point features. To make weight coefficients comparable for different channels, this function consists of shared MLPs followed by a channel-level softmax. It is formulated as

$$
s_{i}^{k}=\mathcal{G}_{s}\left(\mathcal{R}_{1}\left(\hat{\boldsymbol{f}}_{i}, \hat{\boldsymbol{f}}_{i}^{k}\right), \boldsymbol{W}_{s}\right)
$$

where a pairwise function $\mathcal{R}_{1}$ indicates a highlevel relationship between the centroid point and any particular neighbor. Here we define $\mathcal{R}_{1}$ as: $\mathcal{R}_{1}\left(\hat{\boldsymbol{f}}_{i}, \hat{\boldsymbol{f}}_{i}^{k}\right)=\left|\hat{\boldsymbol{f}}_{i}-\hat{\boldsymbol{f}}_{i}^{k}\right|$, which measures the feature difference between point pairs and assigns a higher attention scores to more similar neighbors.

Weighted summation. We consider the learned attention scores as a soft mask that selectively focuses on important features. Then, the local representation $\tilde{\boldsymbol{f}}_{i}$ is obtained by summing weighted features as

$$
\tilde{\boldsymbol{f}}_{i}=\sum_{k=1}^{K}\left\{\hat{\boldsymbol{f}}_{i}^{k} s_{i}^{k}\right\}
$$

\subsection{Position feedback}

Given the extracted features, this module aims to perform feature-guided point shifting. To achieve this, we regress an adaptive $3 \mathrm{D}$ displacement for each point by considering its feature, as well as position. Taking the task loss (cross-entropy loss) as feedback, these displacements can learn to adjust the positions of points. By minimizing intra-class variances (i.e., minimizing feature difference of intra-class points), local points are shifted towards a certain distribution that fits the network well. With respect to the original irregular points, this distribution is more regular and leads to more effective feature learning. In detail, this module comprises the following steps.

\subsubsection{D displacement}

Using dilated KNN, we index $K$ neighboring points of the center point $p_{i}$ as $\left\{p_{i}^{1} \ldots p_{i}^{k} \ldots p_{i}^{K}\right\}$, together with corresponding point features $\left\{\tilde{\boldsymbol{f}}_{i}^{1} \ldots \tilde{\boldsymbol{f}}_{i}^{k} \ldots \tilde{\boldsymbol{f}}_{i}^{K}\right\}$. For each one, we define a dual relation between spatial and semantic levels to generate a point-wise $3 \mathrm{D}$ displacement, formulated as

$$
d_{i}^{k}=\mathcal{G}_{o}\left(\left[\mathcal{R}_{\mathrm{spa}}\left(p_{i}, p_{i}^{k}\right), \mathcal{R}_{\mathrm{sem}}\left(\tilde{\boldsymbol{f}}_{i}, \tilde{\boldsymbol{f}}_{i}^{k}\right)\right], \boldsymbol{W}_{o}\right)
$$

where $\mathcal{R}_{\text {spa }}\left(p_{i}, p_{i}^{k}\right)=\left(p_{i}-p_{i}^{k}\right)$ indicates a spatial relation between point $p_{i}^{k}$ and its center, and $\mathcal{R}_{\text {sem }}\left(\tilde{\boldsymbol{f}}_{i}, \tilde{\boldsymbol{f}}_{i}^{k}\right)=\left|\tilde{\boldsymbol{f}}_{i}-\tilde{\boldsymbol{f}}_{i}^{k}\right|$ is defined using a formulation similar to that for $\mathcal{R}_{1}$. The first term $\mathcal{R}_{\text {spa }}$ ensures that the corresponding point features are always aware of their relative spatial locations, and the second term $\mathcal{R}_{\text {sem }}$ helps to learn shift distance according to feature difference. For each 3D displacement, the former provides direction and the latter, magnitude, which 
jointly guide the irregular neighbors to meaningful patches under the feedback of the task loss.

\subsubsection{Position updating}

The learned adaptive displacements can be viewed as semantics-driven factors. With them, the points are automatically shifted, generally towards a more regular distribution. Formally, each neighboring point is updated as Eq. (6):

$$
\tilde{p}_{i}^{k}=p_{i}^{k}+\alpha \mathcal{N}\left(d_{i}^{k}\right)
$$

where $\alpha$ is a scale coefficient, $\mathcal{N}(\cdot)$ is a normalization function mapping the value to range $[-1,1]$, and $\tilde{p}_{i}^{k}$ are shifted global coordinates. As the network is very fragile at the beginning of training, $\alpha$ is initialized to 0 and gradually assigned an appropriate weight to adapt to local structures.

As shown in Fig. 2, each position feedback module utilizes features extracted from the previous feature modeling module to perform feature-guided point shifting. With iterative downsampling and upsampling, these two modules need to run at different resolutions. We integrate the two consecutive modules operating at the same resolution together into one operation, BidConv, as shown in Fig. 3. We utilize KNN only once after each sampling. Stacking multiple BidConv units enables the two modules to execute alternately and assist each other, thereby forming an effective learning network.

\section{Results and discussion}

\subsection{Datasets}

We evaluated our method on four datasets and multiple tasks ranging from shape classification (ModelNet40 [8]) and part segmentation (ShapeNet Parts [19]) to semantic segmentation (S3DIS [20] and ScanNet [9]). The experimental setting for each dataset is listed below:

- ModelNet40 has 12,311 3D mesh models in 40 object categories. We followed the official split with $9843 / 2468$ models for training and testing, respectively.

- ShapeNet Parts has 16,881 CAD models in 16 object categories. Each point is annotated with a certain one of 50 part classes and each point cloud consists of $2-5$ parts. We followed the official split with with 14,006 objects for training and 2874 for testing.

- ScanNet has 1512 reconstructed indoor scenes in 21 semantic categories. We split this dataset into 1201 and 312 scenes for training and testing, respectively.

- $\quad$ S3DIS has 271 real rooms from three different buildings, in 13 semantic categories. Following the experimental settings in Refs. [12, 36], we used two dominant settings for training and testing: 6-fold and Area-5 cross validation, respectively.

\subsection{Shape classification}

\subsubsection{Experiment}

We evaluated our network on classifying point clouds sampled from ModelNet40 [8]. Using a widelyused sampling density in the existing literature, we uniformly sampled 1024 points from each 3D mesh model and normalized them to a unit sphere. We used overall accuracy (OA) as the evaluation metric. To reduce over-fitting, we employed the dropout technique [44] with $80 \%$ ratio in the penultimate FC layers.

\subsubsection{Results}

For fair comparison, we present overall shape accuracy as well as input settings in Table 1. BLNet clearly surpasses all previous approaches for various input settings. Specifically, when only using $x y z$ information, BLNet achieves an OA of $93.5 \%$ and outperforms a set of representative methods such as PointNet++ [13] (90.7\%), PointCNN [14] (92.2\%), RSCNN (at a single scale, all that is available at

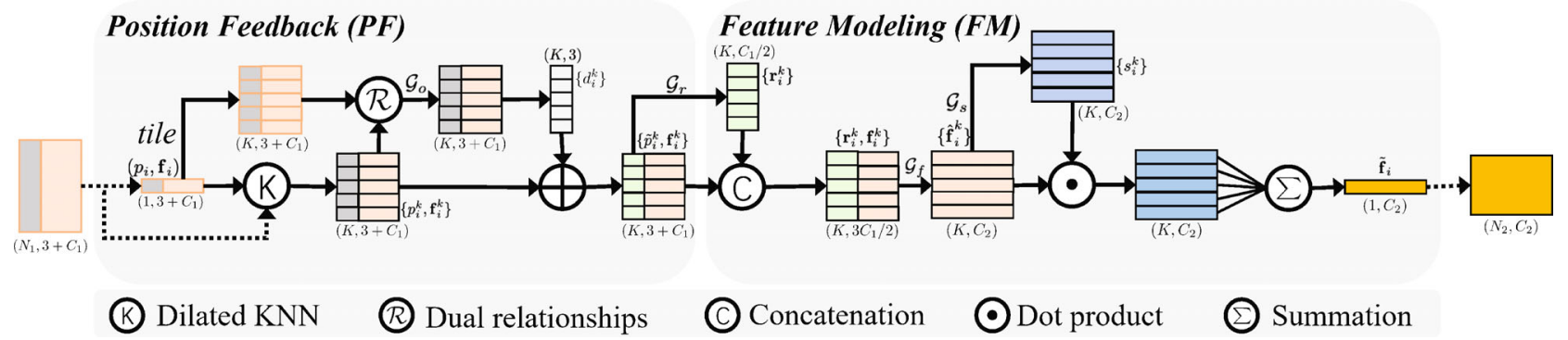

Fig. 3 BidConv with downsampling $\left(N_{2}<N_{1}\right)$. 
Table 1 Overall accuracy using different approaches on ModelNet40

\begin{tabular}{lccc}
\hline Method & Input & \#Points & OA (\%) \\
\hline PointNet [12] & $x y z$ & $1 \mathrm{k}$ & 89.2 \\
PointNet++ [13] & $x y z$ & $1 \mathrm{k}$ & 90.7 \\
KCNet [25] & $x y z$ & $1 \mathrm{k}$ & 91.0 \\
RSCNN [28] & $x y z$ & $1 \mathrm{k}$ & 91.7 \\
DGCNN [16] & $x y z$ & $1 \mathrm{k}$ & 92.2 \\
PointCNN [14] & $x y z$ & $1 \mathrm{k}$ & 92.2 \\
Point2Sequence [37] & $x y z$ & $1 \mathrm{k}$ & 92.6 \\
A-CNN [38] & $x y z$ & $1 \mathrm{k}$ & 92.6 \\
PointASNL [39] & $x y z$ & $1 \mathrm{k}$ & 92.9 \\
Grid-GCN [40] & $x y z$ & $1 \mathrm{k}$ & 93.1 \\
ShellNet [22] & $x y z$ & $1 \mathrm{k}$ & 93.1 \\
InterpCNN [41] & $x y z$ & $1 \mathrm{k}$ & 93.0 \\
Point2Node [42] & $x y z$ & $1 \mathrm{k}$ & 93.0 \\
PCT [29] & $x y z$ & $1 \mathrm{k}$ & 93.2 \\
BLNet (ours) & $\boldsymbol{x y \boldsymbol { z }}$ & $\mathbf{1 k}$ & $\mathbf{9 3 . 5}$ \\
KPConv [26] & $x y z$ & $6 \mathrm{k}$ & 92.9 \\
\hline PointWeb [15] & $x y z$, normal & $1 \mathrm{k}$ & 92.3 \\
PointConv [27] & $x y z$, normal & $1 \mathrm{k}$ & 92.5 \\
BLNet (ours) & $\boldsymbol{x} \boldsymbol{y} \boldsymbol{z}$, normal & $\mathbf{1 k}$ & $\mathbf{9 3 . 7}$ \\
PointNet++ [13] & $x y z$, normal & $5 \mathrm{k}$ & 91.9 \\
SO-Net [43] & $x y z$, normal & $5 \mathrm{k}$ & 93.4 \\
\hline & & &
\end{tabular}

present) [28] (91.7\%), and Point2Node [42] (93.0\%) It also outperforms KPConv [26] (92.9\%) which uses more $(6 \mathrm{k})$ points. Moreover, when additionally using normal information, BLNet achieves a higher accuracy of $93.7 \%$ and significantly outperforms all existing methods, again including PointNet $++[13]$ $(91.9 \%)$ and SO-Net [43] (93.4\%), which use more (5k) points.

\subsection{Part segmentation}

\subsubsection{Experiment}

Part segmentation is a challenging task for finegrained shape analysis. We evaluated our network on ShapeNet Parts [19] benchmark and randomly sampled 2048 points as the input. We report class average $\mathrm{IoU}(\mathrm{mcIoU})$ as the evaluation metric.

\subsubsection{Results}

As in Table 2, BLNet achieves the best performance with an mcIoU of $85.1 \%$. This is considerably better than other competitive baselines: DGCNN [16] (82.3\%), RSCNN [28] (84.0\%), and the recent PointASNL [39] (83.4\%). Figure 4 shows examples for different shapes. BLNet can segment distinctive parts from diverse shapes.
Table 2 Qualitative results (\%) using different approaches on S3DIS, ShapeNet Parts, and ScanNet

\begin{tabular}{lcccccc}
\hline \multirow{2}{*}{ Methods } & \multicolumn{7}{c}{ S3DIS } & 6 -fold & \multicolumn{3}{c}{ S3DIS } & Area-5 & ShapeNet Parts & ScanNet \\
\cline { 2 - 7 } & OA & mIoU & OA & mIoU & mcIoU & OA \\
\hline PointNet [12] & 78.6 & 47.6 & - & 41.1 & 80.4 & 73.9 \\
PointNet++ [13] & 81.0 & 54.5 & - & - & 81.9 & 84.5 \\
KCNet [25] & - & - & - & - & 82.2 & - \\
DGCNN [16] & 84.1 & 56.1 & - & - & 82.3 & - \\
RSCNN [28] & - & - & - & - & 84.0 & - \\
RSNet [45] & - & 56.5 & - & 51.9 & 81.4 & - \\
PointCNN [14] & 88.1 & 65.4 & 85.9 & 57.3 & 84.6 & 85.1 \\
SPGraph [46] & 85.5 & 62.1 & 86.4 & 58.0 & - & - \\
A-CNN [38] & 87.3 & 62.9 & - & - & 83.0 & 85.4 \\
PointWeb [15] & 87.3 & 66.7 & 86.9 & 60.3 & - & 85.9 \\
ShellNet [22] & 87.1 & 66.8 & - & - & 82.8 & 85.2 \\
Point-Edge [47] & 88.2 & 67.8 & 87.2 & 61.8 & - & - \\
ELGS [18] & 87.6 & 66.3 & 88.4 & 60.1 & - & 85.3 \\
Grid-GCN [40] & - & - & 86.9 & 57.8 & - & 85.4 \\
PCT [29] & - & - & - & 61.3 & 83.1 & - \\
PointASNL [39] & 88.8 & 68.7 & 87.7 & 62.6 & 83.4 & - \\
RandLA-Net [48] & 87.2 & 68.5 & - & - & - & - \\
BLNet (ours) & $\mathbf{8 9 . 3}$ & $\mathbf{7 0 . 8}$ & $\mathbf{8 9 . 1}$ & $\mathbf{6 4 . 2}$ & $\mathbf{8 5 . 1}$ & $\mathbf{8 6 . 7}$ \\
\hline
\end{tabular}

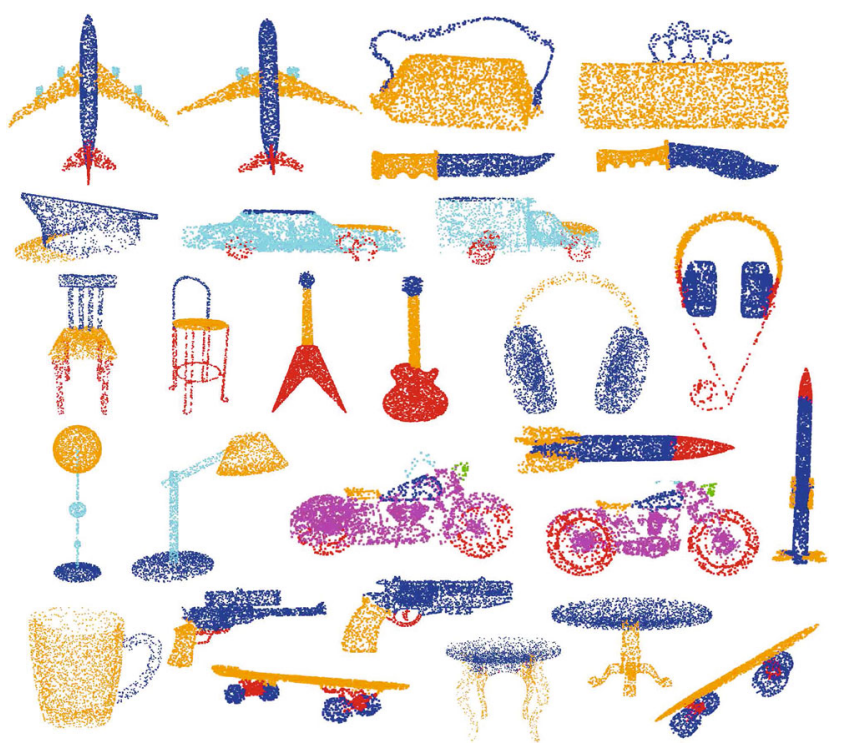

Fig. 4 Part segmentation results from ShapeNet Parts.

\subsection{Semantic segmentation}

\subsubsection{Experiments}

This task takes 3D point clouds as input and assigns one semantic class label to each point. We evaluated our network on two datasets: S3DIS [20] and ScanNet [9]. Following PointCNN [14], we first split points according to room and sliced the rooms into $1.5 \mathrm{~m} \times 1.5 \mathrm{~m}$ blocks, with $0.3 \mathrm{~m}$ padding on each side. We sampled 2048 points for each block 
in training, and adopted all points for evaluation during testing. For S3DIS, we adopted two widelyused evaluation metrics: overall accuracy $(\mathrm{OA})$ and mean class IoU (mIoU). For ScanNet, to make a fair comparison with other approaches, we did not use RGB information and converted the segmentation results from the testing data into semantic voxel labeling for evaluation. Here we report voxel-wise overall accuracy (OA) as the evaluation metric.

\subsubsection{Results on S3DIS}

As shown in Table 2, our BLNet outperforms all previous approaches in terms of the evaluation settings. Specifically, for 6-fold mIoU, BLNet significantly surpasses prior start-of-the-art methods: PointNet $++[13](16.3 \% \uparrow)$, PointCNN [14] $(5.4 \% \uparrow)$, ELGS [18] (4.5\%个), and the recent RandLA-Net [48] (2.3\% $\uparrow$; while for Area-5 mIoU, BLNet considerably outperforms PointNet [12] (23.1\% $\uparrow)$, PointCNN [14 $(6.9 \% \uparrow)$, and also outperforms the recent Grid-GCN [40] (6.4\% $\uparrow)$, PointASNL [39] (1.6\%个). In addition, we list detailed $\mathrm{mIoU}$ results for all the categories in Table 3. BLNet achieves better than or on par performance with other competitive approaches $[12,14,15,18$, $46,48]$. It is worth mentioning that BLNet works well for similar geometric shapes (e.g., column and wall) and the categories with differing shapes (e.g., chair and table). This indicates that BLNet captures discriminative shape representations from complex structures. Some visual results for different real scenes are given in Fig. 5 .

\subsubsection{Results on ScanNet}

As shown in Table 2, when doing semantic voxel labeling, BLNet performs better (86.7\%) than existing methods: PointNet++ [13] (84.5\%),

Table 3 All categories of results (\%) for different approaches on S3DIS 6-fold

\begin{tabular}{|c|c|c|c|c|c|c|c|c|c|c|c|c|c|c|}
\hline Method & $\mathrm{mIoU}$ & ceil. & floor & wall & beam & col. & wind. & door & table & chair & sofa & book. & board & clut. \\
\hline PointNet [12] & 47.6 & 88.0 & 88.7 & 69.3 & 42.4 & 23.1 & 47.5 & 51.6 & 54.1 & 42.0 & 9.6 & 38.2 & 29.4 & 35.2 \\
\hline SPGraph [46] & 62.1 & 89.9 & 95.1 & 76.4 & 62.8 & 47.1 & 55.3 & 68.4 & 69.2 & 73.5 & 45.9 & 63.2 & 8.7 & 52.9 \\
\hline PointCNN [14] & 65.4 & 94.8 & 97.3 & 75.8 & 63.3 & 51.7 & 58.4 & 57.2 & 69.1 & 71.6 & 61.2 & 39.1 & 52.2 & 58.6 \\
\hline ELGS [18] & 66.3 & 93.7 & 95.6 & 76.9 & 42.6 & 46.7 & 63.9 & 69.0 & 70.1 & 76.0 & 52.8 & 57.2 & 54.8 & 62.5 \\
\hline PointWeb [15] & 66.7 & 93.5 & 94.2 & 80.8 & 52.4 & 41.3 & 64.9 & 68.1 & 71.4 & 67.1 & 50.3 & 62.7 & 62.2 & 58.5 \\
\hline RandLA-Net [48] & 68.5 & 92.7 & 95.6 & 79.2 & 61.7 & 47.0 & 63.1 & 67.7 & 68.9 & 74.2 & 55.3 & 63.4 & 63.0 & 58.7 \\
\hline BLNet (ours) & 70.8 & 94.3 & 97.1 & 83.5 & 64.1 & 50.5 & 72.5 & 63.2 & 74.2 & 79.5 & 50.7 & 64.0 & 63.6 & 62.7 \\
\hline
\end{tabular}

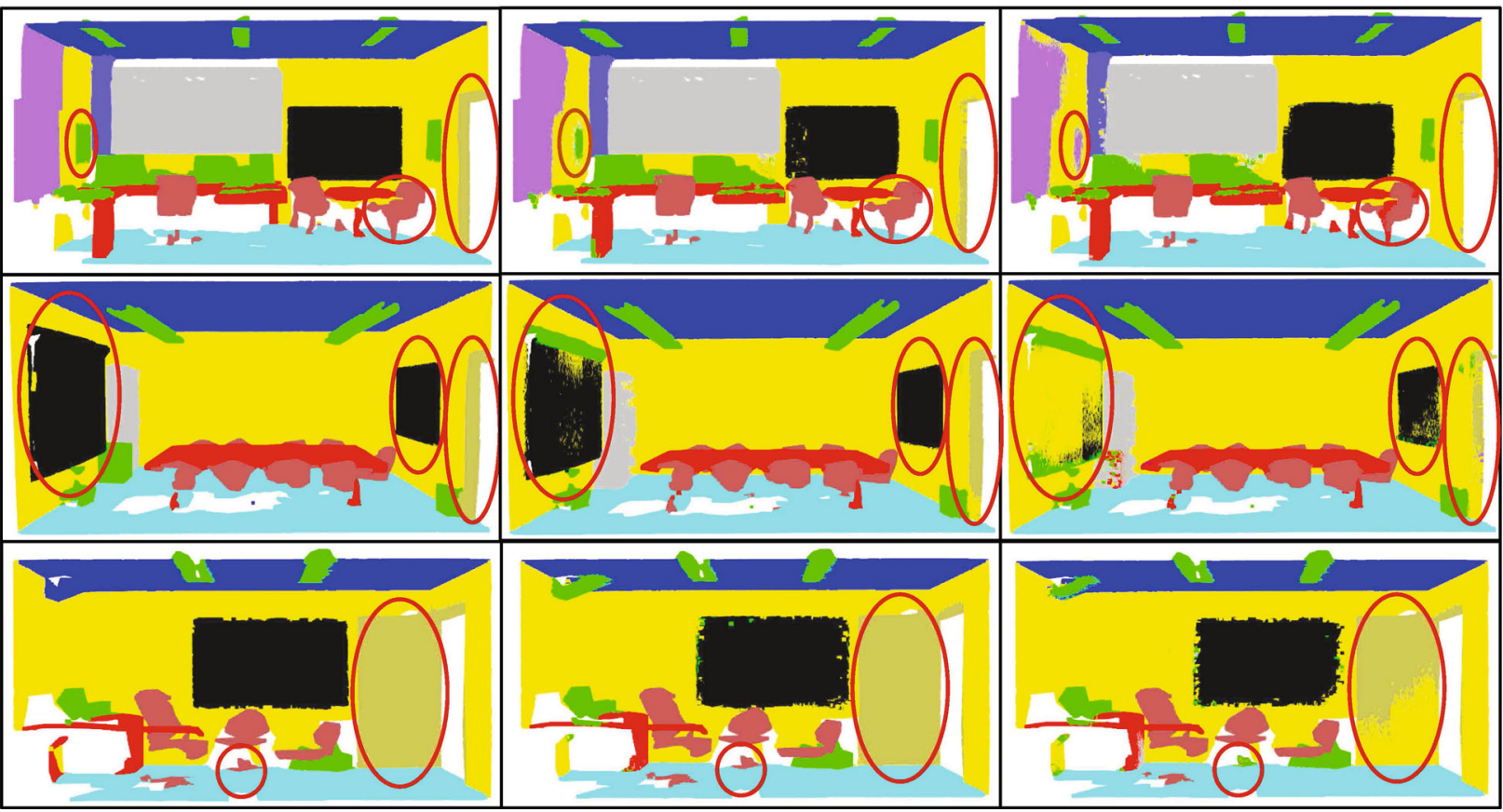

Fig. 5 Results from S3DIS. Left to right: ground truth, BLNet (ours), and PointCNN [14]. Segmentation results of BLNet are closer to the ground truth than those of PointCNN. 
PointCNN [14] (85.1\%), ELGS [18] (85.3\%), and the recent Grid-GCN [40] (85.4\%).

\subsection{Ablation and other studies}

To validate the contribution of each module to our framework, we conducted ablation studies with results in Table 4. These experiments used S3DIS [20] with Area-5 cross validation, with mIoU used as the standard metric. We also considered robustness, model size, and latency.

\subsubsection{Remove position encoding}

Position encoding enables each 3D point to observe its local geometry. After removing this unit, we directly transform point features using per-point MLPs and feed the output features into the subsequent attention pooling. As shown in Table 4, removing position encoding causes a significant performance drop. This is because the spatial distributions of points play a crucial role in 3D shape analysis, and our position encoding unit effectively increases structure-awareness by explicitly encoding relative point positions.

\subsubsection{Replace attention pooling with max/mean pooling}

Attention pooling learns to selectively focus on important features and then combine them. As a comparison, we replaces this unit with the

Table 4 mIoU results for ablated networks based on BLNet

\begin{tabular}{lc}
\hline & mIoU (\%) \\
\hline Remove position encoding & 54.90 \\
Replace with mean-pooling & 58.72 \\
Replace with max-pooling & 60.55 \\
Remove position feedback & 59.70 \\
Full framework (BLNet) & $\mathbf{6 4 . 1 7}$ \\
\hline
\end{tabular}
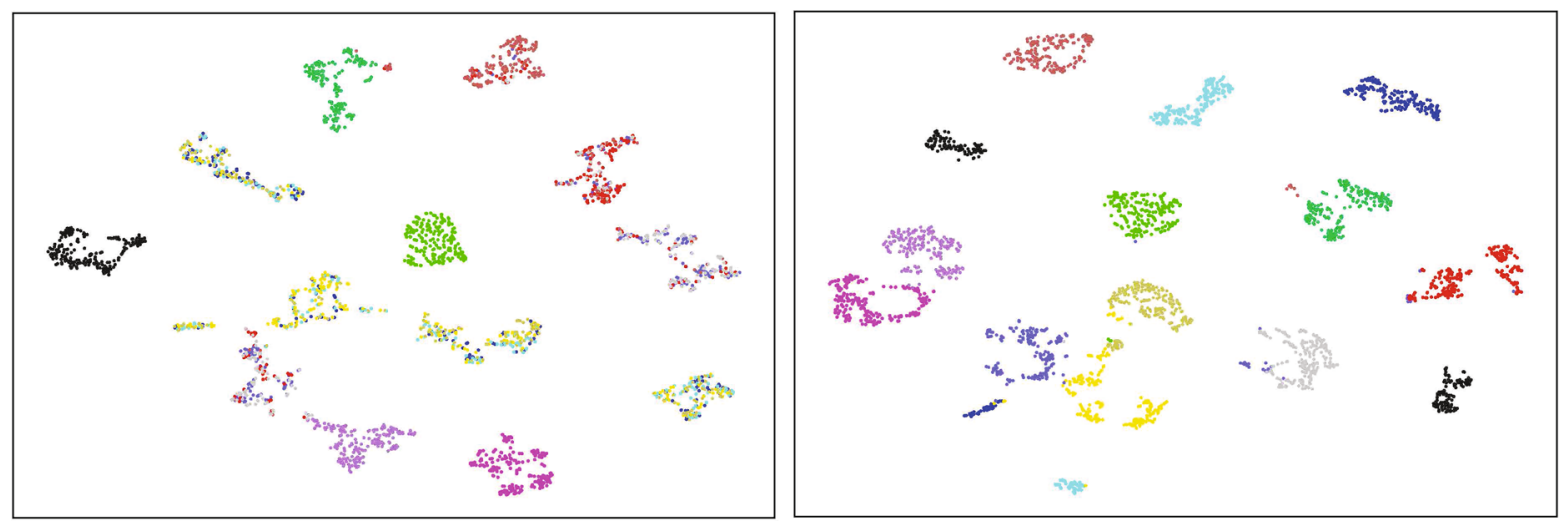

Fig. 6 T-SNE visualization of features without (left) and with (right) position feedback module. Thirteen different colors indicate different categories. Experiments were performed on S3DIS [20]. Left: red, grey, and blue are all mixed; yellow and cyan are mixed. Right: all are clearly separated. widely-used max/mean pooling. As Table 4 shows, our attention pooling considerably improves upon max/mean pooling. This demonstrates that attention pooling is able to keep important features and gather a discriminative representation.

\subsubsection{Remove position feedback}

The position feedback module aims to adaptively shift 3D points towards a more regular distribution. After removing this module, we directly feed original irregular points into the subsequent feature modeling module. As shown in Table 4, removing position feedback considerably harms performance. This indicates that shifted distributions can fit the network better than original ones and promote feature learning. To further verify the effectiveness of position feedback, we visualize T-SNE results of shape features for different categories without and with this module in Fig. 6. Note that since differences in distributions of input points result in different features (see Section 1 Introduction), we visualize features to better view the effect of different input distributions without and with position feedback. In Fig. 6(left), features extracted from irregular points (without position feedback) are mixed and less distinguishable from each other, showing that directly using raw point clouds can cause more shape information loss. In contrast, in Fig. 6(right), features extracted from shifted points (with position feedback) are better partitioned. This shows that shifted distributions induced by adaptive 3D displacements can lead to more intra-class consistency and interclass distinctiveness with respect to the original irregular distributions. 


\subsubsection{Robustness to noise}

We next demonstrate the robustness of our BLNet with respect to two representative baselines, PointNet++ [13] and PointCNN [14]. When applying random noise (increasing irregularity) in the range $[-0.01,0.01]$ to each point, the mIoUs of BLNet, PointCNN, and PointNet++ on S3DIS Area-5 [20] decrease by $1.2 \%, 3.4 \%$, and $4.5 \%$, respectively: BLNet is more robust to random noise. This is because the position feedback module generates adaptive 3D displacements to shift each point, which can be effective in resisting random noise.

\subsubsection{Model size and latency}

Figure 7 qualitatively compares model size for different approaches in terms of accuracy versus parameter memory requirements, as well as comparing accuracy versus latency. Compared to modern competitive methods [12-14, 16, 22, 42], BLNet achieves a significantly better accuracy versus complexity trade-off, demonstrating its effectiveness and efficiency. Note that the BLNet model with the highest accuracy (middle) is our original version, and we increase or decrease its model size by simply scaling feature channels in Fig. 2.

\section{Conclusions}

This is the first work to apply bidirectional learning to point clouds, with bidirectional interaction between points and features. Specifically, we propose a novel point-based bidirectional learning network (BLNet) to analyze irregular 3D points. BLNet optimizes learning for 3D points through two iterative methods: feature-guided point shifting and feature learning from shifted points. The position feedback module

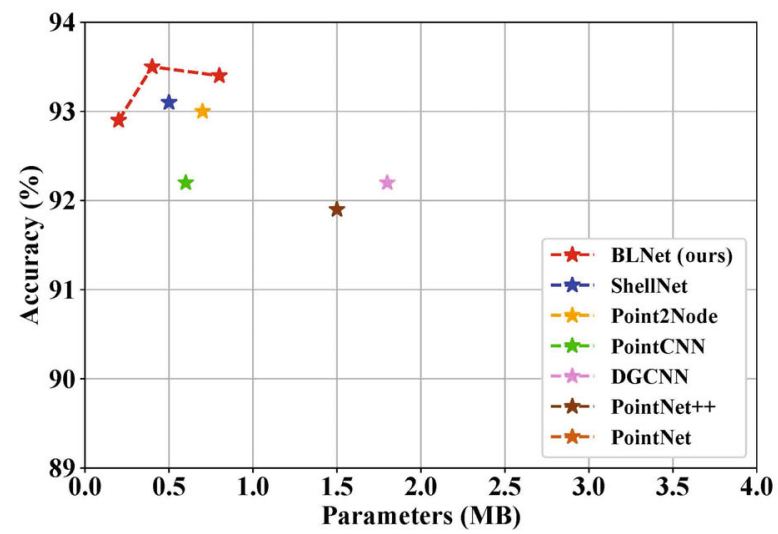

utilizes adaptive 3D displacements to automatically shift points, leading to a more regular distribution. A new feature modeling module explicitly encodes point positions with increased structure-awareness, and a powerful attention pooling in this module selectively combines important features. These two modules alternately regularize the point cloud and learn its geometric features, and iteratively assist each other for more effective feature learning. Extensive experiments have verified the superiority of our BLNet on various challenging benchmarks.

\section{Acknowledgements}

This work was supported by the National Natural Science Foundation of China (Grant No. 62171393), and National Key R\&D Program of China (Grant No. 2021YFF0704600).

\section{Declaration of competing interest}

The authors have no competing interests to declare that are relevant to the content of this article.

\section{References}

[1] Szegedy, C.; Liu, W.; Jia, Y. Q.; Sermanet, P.; Reed, S.; Anguelov, D.; Erhan, D.; Vanhoucke, V.; Rabinovich, A. Going deeper with convolutions. In: Proceedings of the IEEE Conference on Computer Vision and Pattern Recognition, 1-9, 2015.

[2] Simonyan, K.; Zisserman, A. Very deep convolutional networks for large-scale image recognition. arXiv preprint arXiv:1409.1556, 2014.

[3] He, K. M.; Zhang, X. Y.; Ren, S. Q.; Sun, J. Deep residual learning for image recognition. In: Proceedings of the IEEE Conference on Computer Vision and Pattern Recognition, 770-778, 2016.

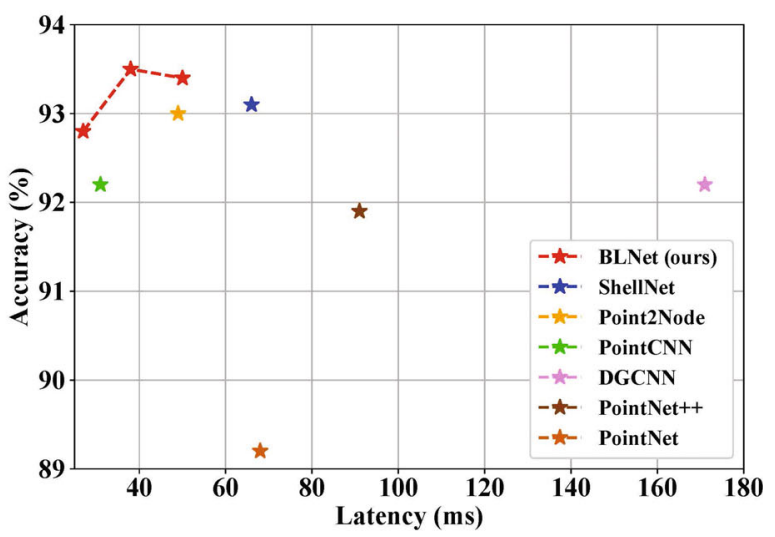

Fig. 7 Comparison between BLNet and other representative baselines on ModelNet40 [8]. Left: accuracy vs. parameters. Right: accuracy vs. latency. All baselines were evaluated on ModelNet40 with batch size 16 and 1024 input points for a fair comparison. 
[4] Qi, C. R.; Su, H.; Nießner, M.; Dai, A.; Yan, M. Y.; Guibas, L. J. Volumetric and multi-view CNNs for object classification on 3D data. In: Proceedings of the IEEE Conference on Computer Vision and Pattern Recognition, 5648-5656, 2016.

[5] Su, H.; Maji, S.; Kalogerakis, E.; Learned-Miller, E. Multi-view convolutional neural networks for 3D shape recognition. In: Proceedings of the IEEE International Conference on Computer Vision, 945-953, 2015.

[6] Kalogerakis, E.; Averkiou, M.; Maji, S.; Chaudhuri, S. $3 \mathrm{D}$ shape segmentation with projective convolutional networks. In: Proceedings of the IEEE Conference on Computer Vision and Pattern Recognition, 6630-6639, 2017.

[7] Maturana, D.; Scherer, S. VoxNet: A 3D Convolutional Neural Network for real-time object recognition. In: Proceedings of the IEEE/RSJ International Conference on Intelligent Robots and Systems, 922-928, 2015.

[8] Wu, Z. R.; Song, S. R.; Khosla, A.; Yu, F.; Zhang, L. G.; Tang, X. O.; Xiao, J. 3D ShapeNets: A deep representation for volumetric shapes. In: Proceedings of the IEEE Conference on Computer Vision and Pattern Recognition, 1912-1920, 2015.

[9] Dai, A.; Chang, A. X.; Savva, M.; Halber, M.; Funkhouser, T.; Nießner, M. ScanNet: Richlyannotated 3D reconstructions of indoor scenes. In: Proceedings of the IEEE Conference on Computer Vision and Pattern Recognition, 2432-2443, 2017.

[10] Klokov, R.; Lempitsky, V. Escape from cells: Deep kdnetworks for the recognition of 3D point cloud models. In: Proceedings of the IEEE International Conference on Computer Vision, 863-872, 2017.

[11] Riegler, G.; Ulusoy, A. O.; Geiger, A. OctNet: Learning deep 3D representations at high resolutions. In: Proceedings of the IEEE Conference on Computer Vision and Pattern Recognition, 6620-6629, 2017.

[12] Charles, R. Q.; Hao, S.; Mo, K. C.; Guibas, L. J. PointNet: Deep learning on point sets for 3D classification and segmentation. In: Proceedings of the IEEE Conference on Computer Vision and Pattern Recognition, 77-85, 2017.

[13] Qi, C. R.; Yi, L.; Su, H.; Guibas, L. J. PointNet++: Deep hierarchical feature learning on point sets in a metric space. In: Proceedings of the 31st International Conference on Neural Information Processing Systems, 5105-5114, 2017.

[14] Li, Y. Y.; Bu, R.; Sun, M. C.; Wu, W.; Di, X. H.; Chen, B. Q. PointCNN: Convolution on X-transformed points. In: Proceedings of the 32nd Conference on Neural Information Processing Systems, 2018.
[15] Zhao, H. S.; Jiang, L.; Fu, C. W.; Jia, J. Y. PointWeb: Enhancing local neighborhood features for point cloud processing. In: Proceedings of the IEEE/CVF Conference on Computer Vision and Pattern Recognition, 5560-5568, 2019.

[16] Wang, Y.; Sun, Y. B.; Liu, Z. W.; Sarma, S. E.; Bronstein, M. M.; Solomon, J. M. Dynamic graph CNN for learning on point clouds. ACM Transactions on Graphics Vol. 38, No. 5, Article No. 146, 2019.

[17] Liu, Z. J.; Tang, H. T.; Lin, Y. J.; Han, S. Point-voxel CNN for efficient 3D deep learning. In: Proceedings of the 33rd Conference on Neural Information Processing Systems, 2019.

[18] Wang, X.; He, J. M.; Ma, L. Exploiting local and global structure for point cloud semantic segmentation with contextual point representations. In: Proceedings of the 33rd International Conference on Neural Information Processing Systems, Article No. 411, 4571-4581, 2019.

[19] Yi, L.; Kim, V. G., Ceylan, D., Shen, I. C., Yan, M. Y.; Su, H.; Lu, A.; Huang, Q.; Sheffer, A. A scalable active framework for region annotation in $3 \mathrm{D}$ shape collections. ACM Transactions on Graphics Vol. 35, No. 6, Article No. 210, 2016.

[20] Armeni, I.; Sener, O.; Zamir, A. R.; Jiang, H.; Brilakis, I.; Fischer, M.; Savarese, S. 3D semantic parsing of large-scale indoor spaces. In: Proceedings of the IEEE Conference on Computer Vision and Pattern Recognition, 1534-1543, 2016.

[21] Jiang, M. Y.; Wu, Y. R.; Lu, C. W. PointSIFT: A SIFT-like network module for 3D point cloud semantic segmentation. arXiv preprint arXiv:1807.00652, 2018.

[22] Zhang, Z. Y.; Hua, B. S.; Yeung, S. K. ShellNet: Efficient point cloud convolutional neural networks using concentric shells statistics. In: Proceedings of the IEEE/CVF International Conference on Computer Vision, 1607-1616, 2019.

[23] Wang, L.; Huang, Y. C.; Hou, Y. L.; Zhang, S. M.; Shan, J. Graph attention convolution for point cloud semantic segmentation. In: Proceedings of the IEEE/CVF Conference on Computer Vision and Pattern Recognition, 10288-10297, 2019.

[24] Kipf, T. N.; Welling, M. Semi-supervised classification with graph convolutional networks. arXiv preprint arXiv:1609.02907, 2016.

[25] Shen, Y. R.; Feng, C.; Yang, Y. Q.; Tian, D. Mining point cloud local structures by kernel correlation and graph pooling. In: Proceedings of the IEEE/CVF Conference on Computer Vision and Pattern Recognition, 4548-4557, 2018.

[26] Thomas, H.; Qi, C. R.; Deschaud, J. E.; Marcotegui, B.; Goulette, F.; Guibas, L. KPConv: Flexible and 
deformable convolution for point clouds. In: Proceedings of the IEEE/CVF International Conference on Computer Vision, 6410-6419, 2019.

[27] Wu, W. X.; Qi, Z. A.; Li, F. X. PointConv: Deep convolutional networks on $3 \mathrm{D}$ point clouds. In: Proceedings of the IEEE/CVF Conference on Computer Vision and Pattern Recognition, 9613-9622, 2019.

[28] Liu, Y. C.; Fan, B.; Xiang, S. M.; Pan, C. H. Relationshape convolutional neural network for point cloud analysis. In: Proceedings of the IEEE/CVF Conference on Computer Vision and Pattern Recognition, 88878896, 2019.

[29] Guo, M. H.; Cai, J. X.; Liu, Z. N.; Mu, T. J.; Martin, R. R.; Hu, S. M. PCT: Point cloud transformer. Computational Visual Media Vol. 7, No. 2, 187-199, 2021.

[30] Engel, N.; Belagiannis, V.; Dietmayer, K. Point transformer. IEEE Access Vol. 9, 134826-134840, 2021.

[31] He, D.; Xia, Y.; Qin, T.; Wang, L.; Yu, N.; Liu, T.; Ma, W. Dual learning for machine translation. In: Proceedings of the 30th International Conference on Neural Information Processing Systems, 820-828, 2016.

[32] Niu, X.; Denkowski, M.; Carpuat, M. Bi-directional neural machine translation with synthetic parallel data. In: Proceedings of the 2nd Workshop on Neural Machine Translation and Generation, 84-91, 2018.

[33] Pontes-Filho, S.; Liwicki, M. Bidirectional learning for robust neural networks. In: Proceedings of the International Joint Conference on Neural Networks, $1-8,2019$

[34] Russo, P.; Carlucci, F. M.; Tommasi, T.; Caputo, B. From source to target and back: Symmetric bidirectional adaptive GAN. In: Proceedings of the IEEE/CVF Conference on Computer Vision and Pattern Recognition, 8099-8108, 2018.

[35] Lin, T. Y.; Dollár, P.; Girshick, R.; He, K. M.; Hariharan, B.; Belongie, S. Feature pyramid networks for object detection. In: Proceedings of the IEEE Conference on Computer Vision and Pattern Recognition, 936-944, 2017.

[36] Tchapmi, L.; Choy, C.; Armeni, I.; Gwak, J.; Savarese, S. SEGCloud: Semantic segmentation of 3D point clouds. In: Proceedings of the International Conference on 3D Vision, 537-547, 2017.

[37] Liu, X. H.; Han, Z. Z.; Liu, Y. S.; Zwicker, M. Point2Sequence: Learning the shape representation of $3 \mathrm{D}$ point clouds with an attention-based sequence to sequence network. Proceedings of the AAAI Conference on Artificial Intelligence Vol. 33, 8778-8785, 2019.

[38] Komarichev, A.; Zhong, Z. C.; Hua, J. A-CNN: Annularly convolutional neural networks on point clouds. In: Proceedings of the IEEE/CVF Conference on Computer Vision and Pattern Recognition, 74137422, 2019

[39] Yan, X.; Zheng, C. D.; Li, Z.; Wang, S.; Cui, S. G. PointASNL: Robust point clouds processing using nonlocal neural networks with adaptive sampling. In: Proceedings of the IEEE/CVF Conference on Computer Vision and Pattern Recognition, 5588-5597, 2020.

[40] Xu, Q. G.; Sun, X. D.; Wu, C. Y.; Wang, P. Q.; Neumann, U. Grid-GCN for fast and scalable point cloud learning. In: Proceedings of the IEEE/ CVF Conference on Computer Vision and Pattern Recognition, 5660-5669, 2020.

[41] Mao, J. G.; Wang, X. G.; Li, H. S. Interpolated convolutional networks for $3 \mathrm{D}$ point cloud understanding. In: Proceedings of the IEEE/CVF International Conference on Computer Vision, 1578-1587, 2019.

[42] Han, W. K.; Wen, C. L.; Wang, C.; Li, X.; Li, Q. Point2Node: Correlation learning of dynamic-node for point cloud feature modeling. Proceedings of the AAAI Conference on Artificial Intelligence Vol. 34, No. 7, 10925-10932, 2020.

[43] Li, J. X.; Chen, B. M.; Lee, G. H. SO-Net: Self-organizing network for point cloud analysis. In: Proceedings of the IEEE/CVF Conference on Computer Vision and Pattern Recognition, 9397-9406, 2018.

[44] Srivastava, N.; Hinton, G.; Krizhevsky, A.; Sutskever, I.; Salakhutdinov, R. Dropout: A simple way to prevent neural networks from overfitting. Journal of Machine Learning Research Vol. 15, 1929-1958, 2014.

[45] Huang, Q. G.; Wang, W. Y.; Neumann, U. Recurrent slice networks for 3D segmentation of point clouds. In: Proceedings of the IEEE/CVF Conference on Computer Vision and Pattern Recognition, 2626-2635, 2018.

[46] Landrieu, L.; Simonovsky, M. Large-scale point cloud semantic segmentation with superpoint graphs. In: Proceedings of the IEEE/CVF Conference on Computer Vision and Pattern Recognition, 4558-4567, 2018.

[47] Jiang, L.; Zhao, H. S.; Liu, S.; Shen, X. Y.; Fu, C. W.; Jia, J. Y. Hierarchical point-edge interaction network for point cloud semantic segmentation. In: Proceedings of the IEEE/CVF International Conference on Computer Vision, 10432-10440, 2019.

[48] Hu, Q. Y.; Yang, B.; Xie, L. H.; Rosa, S.; Guo, Y. L.; Wang, Z. H.; Trigoni, N.; Markham, A. RandLA-net: Efficient semantic segmentation of large-scale point clouds. In: Proceedings of the IEEE/CVF Conference on Computer Vision and Pattern Recognition, 1110511114, 2020. 


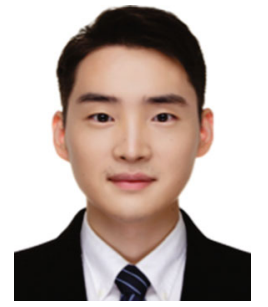

Wenkai Han received his B.Eng. degree in computer science from Xiamen University, China, in 2018. $\mathrm{He}$ is currently working towards his M.Sc. degree in the School of Information Science and Engineering, Xiamen University. His research interests include computer vision, machine learning, and $3 \mathrm{D}$ point cloud processing.

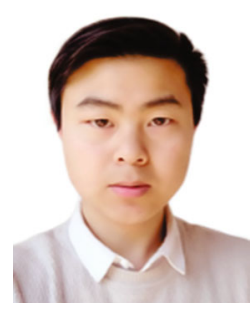

Hai Wu received his B.Eng. degree in information computing and science from Sichuan University of Science and Technology, China, in 2018. He is currently working towards his M.Sc. degree in the School of Informatics, Xiamen University. His research interests include computer vision, machine learning, and 3D point cloud processing.

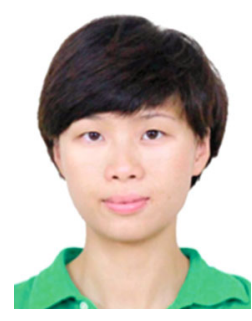

Chenglu Wen received her Ph.D. degree in mechanical engineering from China Agricultural University, Beijing, China, in 2009. She is currently an associate professor with the School of Informatics, Xiamen University. Her current research interests include 3D vision, 3D point cloud processing, and intelligent robots. She has coauthored about 80 research papers and is currently an associate editor of IEEE-GRSL and IEEE T-ITS.

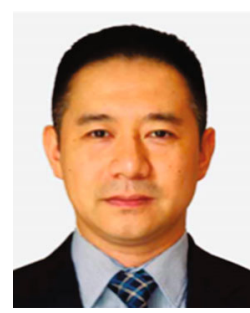

Cheng Wang received his Ph.D. degree in information and communication engineering from the National University of Defense Technology, Changsha, China in 2002. He is currently a professor and associate dean of the School of Informatics, and director of Fujian Key Laboratory of Sensing and Computing for Smart Cities, Xiamen University. His current research interests include 3D vision, LiDAR data analysis, and multisensor fusion. He chaired the ISPRS Working Group I/6 on Multi-sensor Integration and Fusion (2016-2020), and is a council member of the China Society of Image and Graphics. He has coauthored over 150 papers.

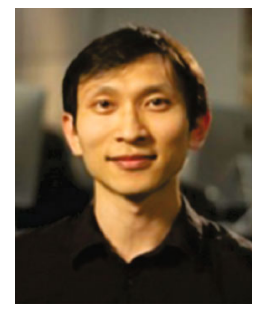

Xin Li received his B.S. degree in computer science from the University of Science and Technology of China in 2003, and his M.S. and Ph.D. degrees in computer science from Stony Brook University (SUNY) in 2008. He is currently a professor with the School of Electrical Engineering and Computer Science and the Center for Computation and Technology, Louisiana State University (LSU), USA. He leads the Geometric and Visual Computing Laboratory at LSU. His research interests include geometric and visual data processing and analysis, computer graphics, and computer vision.

Open Access This article is licensed under a Creative Commons Attribution 4.0 International License, which permits use, sharing, adaptation, distribution and reproduction in any medium or format, as long as you give appropriate credit to the original author(s) and the source, provide a link to the Creative Commons licence, and indicate if changes were made.

The images or other third party material in this article are included in the article's Creative Commons licence, unless indicated otherwise in a credit line to the material. If material is not included in the article's Creative Commons licence and your intended use is not permitted by statutory regulation or exceeds the permitted use, you will need to obtain permission directly from the copyright holder.

To view a copy of this licence, visit http:// creativecommons.org/licenses/by/4.0/.

Other papers from this open access journal are available free of charge from http://www.springer.com/journal/41095. To submit a manuscript, please go to https://www. editorialmanager.com/cvmj. 